Table 1. Efficacy endpoints at Week 16 and Week 52 in TNFi-naïve patients with axSpA

\begin{tabular}{|c|c|c|c|c|}
\hline Endpoints & Week & $\begin{array}{l}\text { Any secuki- } \\
\text { numab } 150 \mathrm{mg} \\
(\mathrm{N}=932)\end{array}$ & $\begin{array}{c}\text { Secukinumab } \\
\begin{array}{c}\mathbf{3 0 0} \mathrm{mg} \\
(\mathrm{N}=\mathbf{5 7})\end{array}\end{array}$ & $\begin{array}{l}\text { Placebo } \\
(\mathrm{N}=569)\end{array}$ \\
\hline \multirow[t]{2}{*}{ ASAS40, n (\%) } & 16 & $399(42.8)^{\dagger}$ & $25(43.9)^{\dagger}$ & $134(23.6)$ \\
\hline & 52 & $508(54.5)$ & 33 (57.9) & - \\
\hline \multirow[t]{2}{*}{ ASAS $5 / 6, n(\%)$} & 16 & $414(44.4)^{\dagger}$ & $24(42.1)^{\dagger}$ & $125(22.0)$ \\
\hline & 52 & $508(54.5)$ & 33 (57.9) & - \\
\hline \multirow[t]{2}{*}{ ASAS partial remission, $\mathrm{n}(\%)$} & 16 & $166(17.8)^{\dagger}$ & $12(21.1)^{\dagger}$ & $35(6.2)$ \\
\hline & 52 & $240(25.8)$ & $13(22.8)$ & - \\
\hline \multirow[t]{2}{*}{ ASDAS-CRP major improvement, $\mathrm{n}(\%)$} & 16 & $266(28.5)^{\dagger}$ & $16(28.1)^{\dagger}$ & $43(7.6)$ \\
\hline & 52 & 336 (36.1) & $20(35.1)$ & - \\
\hline \multirow[t]{2}{*}{ ASDAS-CRP ID, n (\%) } & 16 & $171(18.3)^{\dagger}$ & $11(19.3)^{\dagger}$ & $31(5.4)$ \\
\hline & 52 & 247 (26.5) & $13(22.8)$ & - \\
\hline \multirow[t]{2}{*}{ BASDAI50, n (\%) } & 16 & $356(38.2)^{\dagger}$ & $22(38.6)^{\S}$ & $110(19.3)$ \\
\hline & 52 & 459 (49.2) & 27 (47.4) & - \\
\hline \multirow[t]{2}{*}{$\begin{array}{l}\text { Nocturnal back pain, mean change from } \\
\text { baseline } \pm S E\end{array}$} & 16 & $-31.82 \pm 0.87$ & $-37.99 \pm 3.56^{\dagger}$ & $\begin{array}{c}-18.12 \\
\pm 1.13\end{array}$ \\
\hline & $52^{*}$ & $-41.31 \pm 27.58$ & $-46.56 \pm 24.30$ & - \\
\hline \multirow[t]{2}{*}{$\begin{array}{l}\text { Overall level of morning stiffness, mean } \\
\text { change from baseline } \pm \text { SE }\end{array}$} & 16 & $-3.23 \pm 0.09^{\dagger}$ & $-3.62 \pm 0.35^{\dagger}$ & $\begin{array}{c}-1.95 \pm \\
0.11^{\dagger}\end{array}$ \\
\hline & $52^{*}$ & $-4.24 \pm 2.75$ & $-4.71 \pm 2.74$ & - \\
\hline
\end{tabular}

Data presented as NRI. ${ }^{\dagger} P<0.001,{ }^{\S} P<0.01$ vs placebo; ${ }^{*}$ mean change \pm SD.ASAS, Assessment of SpondyloArthritis international Society; ASDAS, Ankylosing Spondylitis Disease Activity Score; axSpA, axial spondyloarthritis; BASDAI, Bath Ankylosing Spondylitis Disease Activity Index; CRP, C-reactive protein; ID, inactive disease; NRI, non-responder imputation; SD, standard deviation; SE, standard error; TNFi, tumour necrosis factor inhibitor.

Disclosure of Interests: Marina Magrey Consultant of: Novartis, Pfizer, Abbvie, UCB, Eli Lilly, Grant/research support from: Clinical trials with Abbvie and UCB, Jessica A. Walsh Consultant of: AbbVie, Novartis, Eli Lilly and Company, UCB, Grant/research support from: AbbVie, Pfizer, Janssen, feng huang: None declared, Hideto Kameda Speakers bureau: AbbVie, Asahi-Kasei, Astellas, Bristol-Myers Squibb, Chugai, Eisai, Eli Lilly, Gilead Sciences, Janssen, Kyowa Kirin, Mitsubishi-Tanabe, Novartis, Pfizer and UCB., Consultant of: AbbVie, Asahi-Kasei, Astellas, Bristol-Myers Squibb, Chugai, Eisai, Eli Lilly, Gilead Sciences, Janssen, Kyowa Kirin, Mitsubishi-Tanabe, Novartis, Pfizer and UCB., Grant/ research support from: AbbVie, Asahi-Kasei, Astellas, Behringer, Chugai, Eisai, Mitsubishi-Tanabe, Novartis, Jianyuan Wang Employee of: Novartis, Christopher Herrem Employee of: Novartis, Patricia Pertel Employee of: Novartis, Helena Marzo-Ortega Speakers bureau: AbbVie, Celgene, Janssen, Eli Lilly and Company, Novartis, Pfizer, Takeda, and UCB., Consultant of: AbbVie, Celgene, Janssen, Eli Lilly and Company, Novartis, Pfizer, Takeda, and UCB., Grant/research support from: Janssen, Novartis

DOI: 10.1136/annrheumdis-2021-eular.2005

\section{POS0926 NORMALIZATION OF HIGH SENSITIVITY CRP VERSUS CLINICAL RESPONSE TO IXEKIZUMAB AT WEEK 16 IN PATIENTS WITH RADIOGRAPHIC \& NON-RADIOGRAPHIC AXIAL SPONDYLOARTHRITIS: RESULTS FROM THE COAST STUDIES}

H. Marzo-Ortega ${ }^{1}$, X. Juanola ${ }^{2}$, T. Okano ${ }^{3}$, Y. Schymura ${ }^{4}$, A. Bradley ${ }^{5}$, J. Gerwien ${ }^{5}$, B. Monsberger ${ }^{6}$, S. Liu Leage ${ }^{5}$, D. Aletaha ${ }^{7}$, M. Østergaard ${ }^{8}$. ${ }^{1}$ Leeds Teaching Hospitals NHS Trust \& LIRMM, University of Leeds, NIHR Leeds Biomedical Research Centre, Leeds, United Kingdom; ${ }^{2}$ University Hospital Bellvitge, Rheumatology Service, Barcelona, Spain; ${ }^{3}$ Osaka City University, Graduate School of Medicine, Osaka, Japan; ${ }^{4}$ Eli Lilly and Company, Statistics, Indianapolis, United States of America; ${ }^{5}$ Eli Lilly and Company, Medical Rheumatology, Indianapolis, United States of America; ${ }^{6} E$ Eli Lilly and Company, Dermatology, Indianapolis, United States of America; ${ }^{7}$ Medical University of Vienna, Division of Rheumatology, Wien, Austria; ${ }^{8}$ University of Copenhagen, Department of Clinical Medicine, Copenhagen, Denmark

Background: C-reactive protein (CRP), an objective marker of inflammation, can be used to monitor treatment response to biologics in patients with axial spondyloarthritis (axSpA) in addition to evaluating signs \& symptoms. CRP is not elevated in all patients with active axSpA questioning its validity as a universal biomarker of response. Ixekizumab (IXE) demonstrated efficacy in axSpA treatment irrespective of baseline (BL) CRP levels. However, response to IXE categorized on CRP change from $\mathrm{BL}$ is unknown.

Objectives: To evaluate response to IXE treatment from BL through week (wk) 16 in patients with axSpA categorized according to change in high sensitivity (hs) CRP.

Methods: COAST-V (NCT 02696785),-W (NCT02696798), \& -X (NCT02757352), were phase 3 , multicentre, randomized, controlled trials, investigating the efficacy of IXE $80 \mathrm{mg}$ every 4 \& 2 wks in patients with: r-axSpA naïve to biologic disease-modifying antirheumatic drugs (bDMARDs; COAST-V); or who were inadequate responders/intolerant to tumour necrosis factor inhibitors (TNFi;
COAST-W); or who fulfilled Assessment of SpondyloArthritis International Society (ASAS) criteria for non-radiographic (nr)axSpA (COAST-X).

This post hoc analysis focuses on approved dosing regimens. Depending on $\mathrm{BL}$ and wk 16 hsCRP values, patients were categorised as stable low (hsCRP $\leq 5 \mathrm{mg} / \mathrm{L}$ at $\mathrm{BL} \& \leq 5 \mathrm{mg} / \mathrm{L}$ at wk 16 ), normalized (hsCRP $>5 \mathrm{mg} / \mathrm{L}$ at $\mathrm{BL} \& \leq 5 \mathrm{mg} / \mathrm{L}$ at wk 16) or elevated (hsCRP $>5 \mathrm{mg} / \mathrm{L}$ at wk 16 , irrespective of $\mathrm{BL}$ hsCRP). An absolute cutoff of $5 \mathrm{mg} / \mathrm{L}$ was used as the stratification factor in all COAST studies. Data were analyzed by treatment arm. Each trial was analyzed separately.

For hsCRP subgroups, patient demographics \& other characteristics at BL, as well as trajectory over time for the endpoints ASAS40 \& Bath Ankylosing Spondylitis Disease Activity Index (BASDAI) 50 are described. Non-responder imputation was used for missing values.

Results: In all studies at BL, disease activity \& MRI SPARCC score were higher in normalized \& elevated subgroups compared to the stable low subgroup - $\mathbf{a}$. In COAST-V, ASAS40 \& BASDAI50 response was observed in IXE-treated patients regardless of hsCRP change status by wk 16 vs placebo. The highest ASAS40 \& BASDAI50 response rate was reported in patients with normalized hsCRP - b. Similar findings were observed in COAST-W \& -X

Table 1. Baseline Patient demographics \& other characteristics - ITT population, per CRP subgroup

\begin{tabular}{|c|c|c|c|c|c|c|c|c|c|}
\hline & \multicolumn{3}{|c|}{$\begin{array}{l}\text { COAST-V } \\
\text { (r-axSpA, } \\
\text { bDMARD } \\
\text { naive) }\end{array}$} & \multicolumn{3}{|c|}{$\begin{array}{c}\text { COAST-W } \\
\text { (r-axSpA, inadequate } \\
\text { responders /intolerant } \\
\text { to TNFi) }\end{array}$} & \multicolumn{3}{|c|}{$\begin{array}{c}\text { COAST-X } \\
\text { (non-radiographic } \\
\text { axSpA, bDMARD } \\
\text { naïve) }\end{array}$} \\
\hline & $\begin{array}{c}\text { Stable } \\
\text { low } \\
(n=79)\end{array}$ & $\begin{array}{l}\text { Norma- } \\
\text { lized } \\
(n=80)\end{array}$ & $\begin{array}{c}\text { Elevated } \\
(n=98)\end{array}$ & $\begin{array}{c}\text { Stable } \\
\text { low } \\
(n=58)\end{array}$ & $\begin{array}{l}\text { Norma- } \\
\text { lized } \\
(n=34)\end{array}$ & $\begin{array}{c}\text { Elevated } \\
(n=126)\end{array}$ & $\begin{array}{c}\text { Stable } \\
\text { low } \\
(n=78)\end{array}$ & $\begin{array}{l}\text { Norma- } \\
\text { lized } \\
(n=40)\end{array}$ & $\begin{array}{c}\text { Elevated } \\
(n=81)\end{array}$ \\
\hline \multicolumn{10}{|l|}{ Parameter } \\
\hline Age (years) & $\begin{array}{c}43.7 \\
(12.1)\end{array}$ & $\begin{array}{c}38.9 \\
(10.9)\end{array}$ & $\begin{array}{c}42.7 \\
(12.0)\end{array}$ & $\begin{array}{c}50.4 \\
(13.3)\end{array}$ & $\begin{array}{c}46.1 \\
(13.8)\end{array}$ & $\begin{array}{c}45.7 \\
(12.5)\end{array}$ & $\begin{array}{c}44.0 \\
(12.8)\end{array}$ & $\begin{array}{c}37.2 \\
(14.6)\end{array}$ & $\begin{array}{c}38.7 \\
(12.8)\end{array}$ \\
\hline $\begin{array}{l}\text { Male gender, } \\
\mathrm{n}(\%)\end{array}$ & $\begin{array}{c}63 \\
(79.7)\end{array}$ & $\begin{array}{l}71.0 \\
(88.8)\end{array}$ & $\begin{array}{c}78 \\
(79.6)\end{array}$ & $\begin{array}{l}39.0 \\
(67.2)\end{array}$ & $\begin{array}{c}30 \\
(88.2)\end{array}$ & $\begin{array}{c}109 \\
(86.5)\end{array}$ & $\begin{array}{c}35 \\
(44.9)\end{array}$ & $\begin{array}{c}23 \\
(57.5)\end{array}$ & $\begin{array}{c}34 \\
(42)\end{array}$ \\
\hline $\begin{array}{l}\text { Duration of } \\
\text { symptoms } \\
\text { since axSpA } \\
\text { onset (years) }\end{array}$ & $\begin{array}{c}17.5 \\
(11.2)\end{array}$ & $\begin{array}{l}14.4 \\
(9.3)\end{array}$ & $\begin{array}{l}16.1 \\
(9.9)\end{array}$ & $\begin{array}{c}21.7 \\
(12.9)\end{array}$ & $\begin{array}{l}16.8 \\
(11.6)\end{array}$ & $\begin{array}{r}18.9 \\
(10.9)\end{array}$ & $\begin{array}{l}12.1 \\
(9.9)\end{array}$ & $\begin{array}{l}10.3 \\
(9.7)\end{array}$ & $\begin{array}{l}9.5 \\
(9.0)\end{array}$ \\
\hline $\begin{array}{l}\text { HLA-B27 } \\
\text { positive, } \\
\text { n (\%) }\end{array}$ & $\begin{array}{c}69 \\
(87.3)\end{array}$ & $\begin{array}{l}75.0 \\
(93.8)\end{array}$ & $\begin{array}{c}89 \\
(90.8)\end{array}$ & $\begin{array}{c}31.0 \\
(91.2)\end{array}$ & $\begin{array}{c}101 \\
(80.2)\end{array}$ & $\begin{array}{c}101 \\
(80.2)\end{array}$ & $\begin{array}{c}48 \\
(61.5)\end{array}$ & $\begin{array}{c}31 \\
(77.5)\end{array}$ & $\begin{array}{c}67 \\
(82.7)\end{array}$ \\
\hline $\begin{array}{l}\text { BASDAI Total } \\
\text { Score }\end{array}$ & $\begin{array}{c}6.5 \\
(1.4)\end{array}$ & $\begin{array}{c}6.7 \\
(1.5)\end{array}$ & $\begin{array}{c}7.0 \\
(1.1)\end{array}$ & $\begin{array}{c}7.4 \\
(1.5)\end{array}$ & $\begin{array}{c}7.3 \\
(1.3)\end{array}$ & $\begin{array}{c}7.4 \\
(1.3)\end{array}$ & $\begin{array}{c}6.9 \\
(1.5)\end{array}$ & $\begin{array}{c}7.1 \\
(1.6)\end{array}$ & $\begin{array}{c}7.2 \\
(1.5)\end{array}$ \\
\hline $\begin{array}{l}\text { ASDAS Total } \\
\text { Score }\end{array}$ & $\begin{array}{l}3.1 \\
(0.5)\end{array}$ & $\begin{array}{l}3.9 \\
(0.6)\end{array}$ & $\begin{array}{c}4.2 \\
(0.7)\end{array}$ & $\begin{array}{l}3.5 \\
(0.6)\end{array}$ & $\begin{array}{c}4.3 \\
(0.6)\end{array}$ & $\begin{array}{l}4.4 \\
(0.8)\end{array}$ & $\begin{array}{l}3.2 \\
(0.6)\end{array}$ & $\begin{array}{c}4.2 \\
(0.8)\end{array}$ & $\begin{array}{c}4.2 \\
(0.9)\end{array}$ \\
\hline $\begin{array}{l}\text { Spinal Pain } \\
\text { due to AS }\end{array}$ & $\begin{array}{l}7.0 \\
(1.5)\end{array}$ & $\begin{array}{l}7.0 \\
(1.5)\end{array}$ & $\begin{array}{l}7.5 \\
(1.3)\end{array}$ & $\begin{array}{l}7.8 \\
(1.5)\end{array}$ & $\begin{array}{l}7.7 \\
(1.5)\end{array}$ & $\begin{array}{l}7.9 \\
(1.4)\end{array}$ & $\begin{array}{c}7.2 \\
(1.7)\end{array}$ & $\begin{array}{c}7.5 \\
(1.7)\end{array}$ & $\begin{array}{l}7.5 \\
(1.6)\end{array}$ \\
\hline \multirow[t]{2}{*}{$\begin{array}{l}\text { peripheral } \\
\text { articular } \\
\text { manifesta- } \\
\text { tions (>=1 } \\
\text { TJC or }>=1 \\
\text { SJC) }\end{array}$} & $\begin{array}{c}47 \\
(59.5)\end{array}$ & $\begin{array}{l}40 \\
(50)\end{array}$ & $\begin{array}{l}62( \\
63.3)\end{array}$ & $\begin{array}{c}43 \\
(74.1)\end{array}$ & $\begin{array}{c}24 \\
(70.6)\end{array}$ & $\begin{array}{c}86 \\
(68.3)\end{array}$ & $\begin{array}{c}56 \\
(71.8)\end{array}$ & $\begin{array}{c}33 \\
(82.5)\end{array}$ & $\begin{array}{c}66 \\
(81.5)\end{array}$ \\
\hline & & MRI & I Spine SP & ARCC S & Score & & MRI SI & IJ SPARC & CC Score \\
\hline $\begin{array}{l}\text { MRI SPARCC } \\
\text { Score }\end{array}$ & $\begin{array}{c}6.7 \\
(11.2)\end{array}$ & $\begin{array}{l}21.8 \\
(27.3)\end{array}$ & $\begin{array}{c}20.7 \\
(25.7)\end{array}$ & $\begin{array}{c}1.2 \\
(2.3)\end{array}$ & $\begin{array}{c}7.0 \\
(7.6)\end{array}$ & $\begin{array}{c}10.4 \\
(17.0)\end{array}$ & $\begin{array}{c}5.2 \\
(6.6)\end{array}$ & $\begin{array}{c}6.1 \\
(8.2)\end{array}$ & $\begin{array}{c}6.2 \\
(10.5)\end{array}$ \\
\hline
\end{tabular}

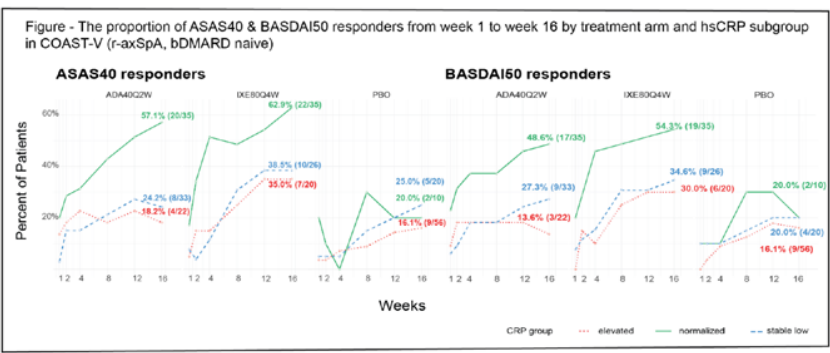

Conclusion: IXE reduced clinical disease activity in patients with axSpA irrespective of hsCRP change from BL to wk 16. Improvement in hsCRP level was associated with overall response rates.

Acknowledgements: The authors would like to acknowledge Philana Fernandes, an employee of Eli Lilly and Company, for her for writing and editorial support. Disclosure of Interests: Helena Marzo-Ortega Consultant of: Celgene, Janssen, El Lilly, Novartis, Pfizer, UCB, Grant/research support from: Janssen, Novartis, Xavie Juanola: None declared, Tadashi Okano Speakers bureau: Asahi Kasei, Astellas, Abbvie, Ayumi, Chugai, Daiichi-Sankyo, Eisai, Eli Lilly, Janssen, Mitsubishi Tanabe, 
Novartis, Ono, Pfizer, Sanofi and Takeda, Grant/research support from: Asahi Kasei, Abbvie, Chugai, Eisai, Yves Schymura Employee of: Eli Lilly, Andrew Bradley Shareholder of: Eli Lilly, Employee of: Eli Lilly, Jens Gerwien Shareholder of: Eli Lilly, Employee of: Eli Lilly, Brigitte Monsberger Shareholder of: Eli Lilly, Employee of: Eli Lilly, Soyi Liu Leage Employee of: Eli Lilly, Daniel Aletaha Speakers bureau: Abbvie, Amgen, Celgene, Lilly, Medac, Merck, Novartis, Pfizer, Roche, Sandoz, Sanofi/Genzyme, Grant/research support from: Abbvie, Lilly, Novartis, Roche, Mikkel Østergaard Speakers bureau: Abbvie, Celgene, Eli-Lilly, Janssen, Novartis, Pfizer, Roche, Sanofi and UCB, Consultant of: Abbvie, BMS, Boehringer-Ingelheim, Celgene, Eli-Lilly, Hospira, Janssen, Merck, Novartis, Novo, Orion, Pfizer, Regeneron, Roche, Sandoz, Sanofi and UCB, Grant/research support from: Abbvie, BMS, Merck, Celgene, Novartis DOI: 10.1136/annrheumdis-2021-eular.2079

\begin{tabular}{|l|l|}
\hline POS0927 & EFFECTIVENESS AND SAFETY OF SECUKINUMAB \\
& IN NAIIVE OR TNF-INHIBITORS FAILURE AXIAL \\
& SPONDYLOARTHRITIS PATIENTS IN REAL LIFE: A \\
& 24-MONTHS PROSPECTIVE MULTICENTRIC STUDY
\end{tabular}

M. Lorenzin ${ }^{1}$, A. Ortolan ${ }^{1}$, M. S. Chimenti ${ }^{2}$, A. Marchesoni ${ }^{3}$, E. Lubrano ${ }^{4}$ L. Santo ${ }^{5}$, A. Semeraro ${ }^{6}$, C. Salvarani ${ }^{7,8}$, N. Girolimetto ${ }^{8}$, E. Praino ${ }^{9}$, G. L. Fonti ${ }^{2}$, R. Foti ${ }^{10}$, A. Carletto ${ }^{11}$, A. Doria ${ }^{12}$, R. Ramonda ${ }^{12}$ On behalf of Italian Society of Rheumatology (SIR) "Spondyloartritis and Psoriatic Arthritis Study Group-A. Spadaro.' ' University of Padova, Rheumatology Unit, Department of Medicine DIMED, Padova, Italy; ${ }^{2}$ University of Rome "Tor Vergata", Rheumatology, Allergology and Clinical Immunology Department of "Medicina dei Sistemi", Roma, Italy; ${ }^{3}$ ASST Gaetano Pini-CTO, Milan, Department of Rheumatology, Milano, Italy; ${ }^{4}$ University of Molise, Campobasso, Academic Rheumatology Unit, Dipartimento di Medicina e Scienze per la Salute "Vincenzo Tiberio", Campobasso, Italy; ${ }^{5}$ ASL BT Andria - DSS4 Barletta, Italy, Barletta-Andria-Trani, Rheumatology Unit, Barletta-Andria-Trani, Italy; ${ }^{6}$ Martina Franca-ASL Taranto, Rheumatology Unit, Taranto, Italy; ${ }^{7}$ University of Modena and Reggio Emilia, Rheumatology Unit, Reggio Emilia, Italy; ${ }^{8}$ Azienda USL-IRCCS, Istituto di Ricovero e Cura a Carattere Scientifico, Rheumatology Unit, Department of Internal Medicine, Reggio Emilia, Italy; ${ }^{9}$ ASL BT Andria - DSS4 Barletta, Italy, Barletta-Andria-Trani, Rheumatology Unit, Barletta-Andria-Trani, Italy; ${ }^{10}$ A. O.U. Policlinico S. Marco, Catania, Italy, Rheumatology Unit, Catania, Italy; ${ }^{11}$ AOUI University of Verona, Rheumatology Unit, Department of Medicine, Verona, Italy; ${ }^{1}$ University of Padova, Rheumatology Unit, Department of Medicine DIMED, Padova, Italy

Background: Axial Spondyloarthritis (axSpA) can be distinguished in radiographic axSpA (r-axSpA) and non-radiographic (nr-axSpA). Secukinumab (SEC) is a novel treatment for axSpA, but data from real-life are still missing.

Objectives: 1)to evaluate the effectiveness and safety of a wide cohort of axSpA patients on SEC followed in 8 Italian Rheumatologic centers for 24-months;2) to compare the features and disease-activity indices of SEC-treated axSpA patients subdivided in naïve biological drugs (group A) and in TNF-inhibitors failure patients (group B).

Methods: Consecutive patients with active axSpA (diagnosis according Assessment of SpondyloArthritis International Society ASAS criteria), who started SEC treatment, were evaluated prospectively.Data on disease characteristics, previous/ongoing treatments and imaging were collected. Disease-activity/functional/ clinical scores and biochemical values were recorded at baseline (T0), at 6 (T6), 12 (T12), and 24 (T24) months. Effectiveness was evaluated over-time with descriptive statistics. Anova (Kruskal Wallis) and generalized linear models were used to compare variables over-time. Infections, adverse events were collected. Results: One-hundred-seven patients [49.53\% men; median age 49years; median treatment duration 18.5years] were enrolled;53(49.53\%) had HLA-B27, 47.66\% were $\mathrm{r}$-axSpA and $52.34 \% \mathrm{nr}$-axSpA. Signs of sacroiliitis were present on MRI in $97(90.65 \%)$ and X-rays in $51(47.66 \%)$. SEC was prescribed as first line biologic treatment in $32(29.9 \%)$ patients and as second or more line biological treatment in $75(70.1 \%)$ patients (Figure 1). In all population significant decrease was achieved in:Visual Analogue Scale of pain and general-health; Leeds Enthesitis Index; Health Assessment Questionnaire modified for spondyloarthritis (HAQ-s);Bath Ankylosing Spondylitis Functional Index (BASFI);C-reactive protein. Bath Ankylosing Spondylitis Metrology Index and Erythrocyte-sedimentation-rate not significantly decreased. Effectiveness was associated to an improvement in Ankylosing Spondylitis disease activity score (ASDAS) [T0=3.4 (2.9-3.9) vs T24=1.9 (1.2-2.7); $\mathrm{p}=0.02]$ and in Bath Ankylosing Spondylitis Disease Activity Index (BASDAI) $[$ T0=6.6 (5.0-7.8) vs T24=3.2 (2.0-5.0); $p=0.03$ ].At T0 group $B$ had a longer disease duration $(p=0.04)$, a greater prevalence of peripheral arthritis $(p=0.02)$,enthesitis $(p=0.04)$ and psoriasis $(p=0.05)$ and was mostly male $(p=0.05)$, while no significant difference was observed for functional and disease-activity indices and signs of sacroilitis on MRI/X-rays. At T24 group A showed better physical functioning and lower disease activity compared to group $B$ [HAQs A vs. $B=0.1(0.0-0.5)$ vs $0.3(0.1-0.8)$; $B A S F I A$ vs $B=1.6(0.8-4.8)$ vs 4.0(2.5-4.6); BASDAI A vs $B=2.2(1.0-3.8)$ vs 3.9(2.7-5.0);ASDAS A vs $B=1.3(1.0-2.2)$ vs 2.1(1.6-2.9)].After T24 of treatment $70.2 \%$ of Group A and $68.4 \%$ of Group B had a low disease activity,accordingly to ASDAS<2.1. Twenty-three patients $(21.5 \%)$ stopped the treatment during the follow-up mainly because of primary (7) or secondary loss of efficacy (9). Only 7 patients suspended SEC because of adverse events.A low number of episodes of mild infections (19) occurred;SEC was instead permanently discontinued in 4 cases for:oral refractory mucositis (2);recurrent aphthosis (1);recurren broncopneumoniae (1).The retention rate at 24 was good in the whole population (73\%).Survival curves for Group A and B were similar (log-rank test=0.81; $=0.69$ ). Conclusion: In a real-life clinical setting,SEC was safe and effective in axSpA, as shown by a significant decrease of BASDAI and ASDAS over a 24-months follow-up.

Baseline characteristics of 107 axSpA patients treated with secukinumab in the period January 2017- December 2020

\begin{tabular}{|c|c|c|c|c|}
\hline axSpA Features & Total patients & GROUP A vs. & GROUP B & p \\
\hline Male sex $(\mathrm{N}, \mathrm{s})$ & $53(49.53 \%)$ & $23(71.88 \%)$ & $30(40 \%)$ & 0.05 \\
\hline Age (years), median (IOR) & $49(40.5-58)$ & $44(35.75-58)$ & $52(42-58.5)$ & 0.04 \\
\hline Age of diagnosis (years), median (IOR) & $43(32.5-51)$ & $39.5(30.5-50)$ & 43 (33.5.53) & ns \\
\hline Age of disease onset (years), median (IQR) & $39(29.5-49)$ & $36.5(22.75-46)$ & $40(30.5-49)$ & ns \\
\hline Disease duration (years), median (IQR) & $9(4-13.5)$ & $7(2-14.25)$ & $10(6-13)$ & 0.04 \\
\hline $\operatorname{axspA}(n, \%)$ & 107 & $32(29.91 \%)$ & 75 (70.09\%) & NA \\
\hline r-axSPA & $51(47.66 \%)$ & $15(46.88 \%)$ & $36(48 \%)$ & ns \\
\hline$n r-a x S \rho A$ & $56(52.34 \%)$ & $17(53.13 \%)$ & $39(52 \%)$ & ns \\
\hline HLA-B27 positive, $\mathrm{N}(\mathbf{\%})$ & $53(49.53 \%)$ & $16(50 \%)$ & $37(49.33 \%)$ & ns \\
\hline SU-MRI positive, $N(\$)$ & $97(90.65 \%)$ & $28(87.5 \%)$ & $69(92 \%)$ & ns \\
\hline Su-X-rays positive, $N(\mathrm{~s})$ & $51(47.66 \%)$ & $15(46.9 \%)$ & $36(48 \%)$ & ns \\
\hline Peripheral arthritis, $\mathrm{N}(\mathrm{s})$ & $62(57.94 \%)$ & $15(46.9 \%)$ & $47(62.67 \%)$ & 0.02 \\
\hline Enthesitis, $N(\mathbf{m})$ & $66(61.68 \%)$ & $17(53.13 \%)$ & $49(65.33 \%)$ & 0.04 \\
\hline Dactylitis, $N$ (\$) & $14(13.08 \%)$ & $5(15.63 \%)$ & $9(12 \%)$ & ns \\
\hline Psoriasis, $\mathbf{N}$ (\%) & $50(46.73 \%)$ & $13(40.63 \%)$ & $37(49.33 \%)$ & 0.05 \\
\hline Onicopathy, N (\%) & $22(20.5656)$ & $6(18.75 \%)$ & $16(21.33 \%)$ & ns \\
\hline $180, N(\%)$ & $4(3.74 \%)$ & $2(6.25 \%)$ & $2(2.67 \%)$ & ns \\
\hline Uveitis, $\mathbf{N}(\%)$ & $5(4.67 \%)$ & $0(0 \%)$ & $5(6.67 \%)$ & ns \\
\hline Familiarity with psoriasis or SpA & $36(33.64 \%)$ & $12(37.5 \%)$ & $24(32 \%)$ & ns \\
\hline Smoking, $N(\%)$ & $26(24.30 \%)$ & $8(25 \%)$ & $18(24 \%)$ & ns \\
\hline Weight (kg), median (IOR) & $70(60.5-82)$ & $70.50(64.5-82.25)$ & $70(60-81.5)$ & ns \\
\hline Height (cm), median (IOR) & $169(161-177)$ & $174(165-178)$ & $168(160-175.5)$ & ns \\
\hline BMI, median (IOR) & $24.69(21.95-28.05)$ & $23.97(22.65-26.20)$ & $24.9(21.79-28.24)$ & ns \\
\hline BASMI [0-10], median (IQR) & $3(1-6)$ & $2(0-4)$ & $4(1-7)$ & 0.03 \\
\hline LE [0-6], median (IOR) & $1(0-2)$ & $0(0-1.5)$ & $1(0-2)$ & ns \\
\hline ESR [0-25](mm/h), median (IOR) & $16(9-28)$ & $13.5(5-27.25)$ & $18.5(10-28)$ & ns \\
\hline CRP $[0-6](\mathrm{mg} / \mathrm{L})$, median (IOR) & $5(2 \cdot 9 \cdot 11)$ & $3.45(2.4-15.3)$ & $5(2.9-11)$ & 0.04 \\
\hline VAS-pain [0-10], median (IOR) & $7(6-8)$ & $6.65(6-7.45)$ & $7(6-8)$ & ns \\
\hline VAS-gh [0-10], median (IOR) & $5.6(4-7)$ & $5.0(4.25-7.0)$ & $6(4-7.1)$ & ns \\
\hline HAQ-S [0-8], median (IOR) & $1(0.5-1.5)$ & $0.75(0.2-1.0)$ & $1(0.75-1.50)$ & 0.05 \\
\hline BASFI [0-10], median (IOR) & $6(4.95-7.03)$ & $5.5(3.63-7.05)$ & $6(5.1-7.0)$ & ns \\
\hline BASDAI [0-10], median (IOR) & $6.6(5-7.8)$ & $6(3.7-6.95)$ & $7(5.25-8.2)$ & ns \\
\hline ASDAS [0-6], median (IOR) & $3.35(2.85-3.86)$ & $3.33(2.67-3.68)$ & $3.35(2.90-3.86)$ & ns \\
\hline Mean treatment duration (months), median (IIR) & $18.5(12-32.5)$ & $18.10(12-30)$ & $18.93(12-32.5)$ & ns \\
\hline Dosage $300 \mathrm{mg} /$ injection $(\mathrm{N}, \mathrm{s})$ & 33 (30.84\%) & $6(18.75 \%)$ & $27(36 \%)$ & 0.04 \\
\hline Dosage $150 \mathrm{mg} /$ /injection $(\mathrm{N}, \%)$ & 74 (69.16\%) & $26(81.25 \%)$ & $48(64 \%)$ & 0.04 \\
\hline $1^{1 " t}$ line $(N, \%)$ & $32(29.91 \%)$ & $32(100 \%)$ & $0(0 \%)$ & NA \\
\hline Failure biological drugs $(\mathrm{N}, \%)$ & $75(70.10 \%)$ & $0(056)$ & $75(100 \%)$ & NA \\
\hline $2^{\text {nd }}$ line $(N, \%)$ & 33 (30.84\%) & 0 (0\%) & $33(44 \%)$ & NA \\
\hline $3^{\text {rt }}$ line $(N, \%)$ & $23(21.50 \%)$ & $0(086)$ & $23(30.67 \%)$ & NA \\
\hline $4^{4 k}$ line $(N, \%)$ & $13(12.15 \%)$ & $0(0 \%)$ & $13(17.33 \%)$ & NA \\
\hline $25^{\mathrm{th}}$ line $(\mathrm{N}, \%)$ & $6(5.61 \%)$ & $0(0 \%)$ & $6(8 \%)$ & NA \\
\hline Concomitant NSAIDS, N (\%) & $68(63.55 \%)$ & $21(65.63 \%)$ & $47(62.67 \%)$ & ns \\
\hline Concomitant glucocorticosteroid, $\mathrm{N}(\%)$ & 22 (20.56\%) & $6(18.75 \%)$ & $16(21.33 \%)$ & ns \\
\hline Concomitant CSDMARDs, $\mathbf{N}(\mathbf{\%})$ & 23 (21.50\%) & $6(18.75 \%)$ & $17(22.67 \%)$ & \\
\hline \multicolumn{5}{|c|}{ 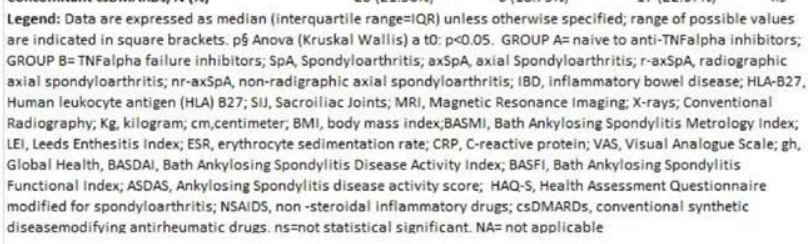 } \\
\hline
\end{tabular}

Disclosure of Interests: Mariagrazia Lorenzin: None declared, Augusta Ortolan: None declared, Maria Sole Chimenti: None declared, Antonio Marchesoni Grant/research support from: AM has received honoraria and speaker fees from Abbvie, Pfizer, MSD, UCB, Novartis, Janssen, Eli-Lilly., Ennio Lubrano: None declared, Leonardo Santo Speakers bureau: Speaker from Jansen, Novartis, Pfizer, UCB, MSD, Sanofi, Angelo Semeraro: None declared, Carlo Salvarani: None declared, Nicolò Girolimetto: None declared, Emanuela Praino: None declared, Giulia Lavinia Fonti: None declared Rosario Foti: None declared, Antonio Carletto: None declared, Andrea Doria Grant/ research support from: ADhas received honoraria and speaker fees from Novartis Abbvie, Pfizer, MSD, Janssen., Roberta Ramonda Grant/research support from: RR has received honoraria and speaker fees from Novartis, Abbvie, Pfizer, MSD, Janssen. DOI: 10.1136/annrheumdis-2021-eular.2121

\section{POS0928 NETAKIMAB EFFICACY IN ANTI-TNF-NAIVE AND ANTI-TNF-EXPERIENCED PATIENTS WITH ACTIVE ANKYLOSING SPONDYLITIS: RESULTS OF SUBANALYSIS OF PHASE 3 ASTERA TRIAL}

S. Erdes ${ }^{1}$, V. Mazurov ${ }^{2}$, T. Dubinina ${ }^{3}$, I. Gaydukova ${ }^{2,4}$, A. Kundzer ${ }^{5}$, N. Soroka ${ }^{6}$, A. Eremeeva ${ }^{7} .{ }^{1}$ Nasonova Research Institute of Rheumatology, Laboratory of Spondyloarthritides, Moscow, Russian Federation; ${ }^{2}$ Mechnikov NorthWestern State Medical University, Department of Therapy and Rheumatology 